\title{
Guideline update for the performance of fusion procedures for degenerative disease of the lumbar spine. Part 13: Injection therapies, low-back pain, and lumbar fusion
}

\author{
William C. Watters III, M.D., ${ }^{1}$ Daniel K. Resnick, M.D., ${ }^{2}$ Jason C. Eck, D.O., M.S., ${ }^{3}$ \\ Zoher Ghogawala, M.D. ${ }^{4}$ Praveen V. Mummaneni, M.D., ${ }^{5}$ Andrew T. Dailey, M.D., ${ }^{6}$ \\ Tanvir F. Choudhri, M.D. .7 Alok Sharan, M.D. ${ }^{8}$ Michael W. Groff, M.D., ${ }^{9}$ \\ Jeffrey C. Wang, M.D., ${ }^{10}$ Sanjay S. Dhall, M.D., ${ }^{5}$ and Michael G. Kaiser, M.D. ${ }^{11}$
}

${ }^{1}$ Bone and Joint Clinic of Houston, Houston, Texas; ${ }^{2}$ Department of Neurosurgery, University of Wisconsin, Madison, Wisconsin; ${ }^{3}$ Center for Sports Medicine and Orthopaedics, Chattanooga, Tennessee; ${ }^{4}$ Alan and Jacqueline Stuart Spine Research Center, Department of Neurosurgery, Lahey Clinic, Burlington, and Tufts University School of Medicine, Boston, Massachusetts; ${ }^{5}$ Department of Neurological Surgery, University of California, San Francisco, California; ${ }^{6}$ Department of Neurosurgery, University of Utah, Salt Lake City, Utah; ${ }^{7}$ Department of Neurosurgery, Icahn School of Medicine at Mount Sinai, New York, New York; ${ }^{8}$ Department of Orthopaedic Surgery, Montefiore Medical Center, Albert Einstein College of Medicine, Bronx, New York; ${ }^{9}$ Department of Neurosurgery, Brigham and Women's Hospital, Boston, Massachusetts; ${ }^{10}$ Department of Orthopaedic Surgery, Keck School of Medicine, University of Southern California, Los Angeles, California; and ${ }^{11}$ Department of Neurosurgery, Columbia University, New York, New York

\begin{abstract}
The medical literature continues to fail to support the use of lumbar epidural injections for long-term relief of chronic back pain without radiculopathy. There is limited support for the use of lumbar epidural injections for shortterm relief in selected patients with chronic back pain. Lumbar intraarticular facet injections are not recommended for the treatment of chronic lower-back pain. The literature does suggest the use of lumbar medial nerve blocks for short-term relief of facet-mediated chronic lower-back pain without radiculopathy. Lumbar medial nerve ablation is suggested for 3-6 months of relief for chronic lower-back pain without radiculopathy. Diagnostic medial nerve blocks by the double-injection technique with an $80 \%$ improvement threshold are an option to predict a favorable response to medial nerve ablation for facet-mediated chronic lower-back pain without radiculopathy, but there is no evidence to support the use of diagnostic medial nerve blocks to predict the outcomes in these same patients with lumbar fusion. There is insufficient evidence to support or refute the use of trigger point injections for chronic lowerback pain without radiculopathy.
\end{abstract}

(http://thejns.org/doi/abs/10.3171/2014.4.SPINE14281)

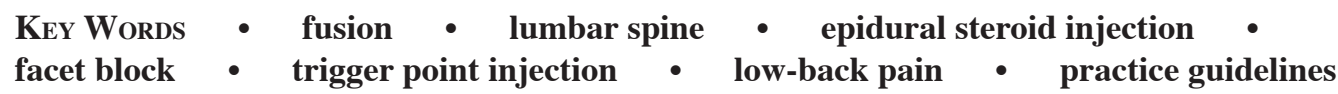

\section{Therapeutic Recommendations}

There is no new evidence that conflicts with the previous recommendations regarding injection therapies published in the original version of the "Guidelines for the performance of fusion procedures for degenerative disease of the lumbar spine." 27

\section{Lumbar Epidural Steroid Injections}

\section{Grade C}

Lumbar epidural steroid injections (ESIs) are an op-

Abbreviations used in this paper: $\mathrm{ESI}=$ epidural steroid injection; NRS = Numerical Rating Scale; ODI = Oswestry Disability Index; $\mathrm{RCT}=$ randomized control trial; $\mathrm{TPI}=$ trigger point injection; VAS = visual analog scale. tion for the short-term relief of chronic low-back pain without radiculopathy in patients with degenerative disease of the lumbar spine (Level III evidence).

Caudal ESIs are an option for decreasing low-back pain of greater than 6 weeks' duration, without radiculopathy, in patients with degenerative disease of the lumbar spine (Level III evidence).

\section{Lumbar Facet Injections}

\section{Grade B}

Intraarticular injections of lumbar facet joints are not suggested for the treatment of facet-mediated chronic low-back pain without radiculopathy in cases of degenerative disease of the lumbar spine (single Level II study and single Level III study).

Lumbar medial nerve blocks are suggested for the 
short-term relief of facet-mediated chronic low-back pain without radiculopathy in patients with degenerative disease of the lumbar spine (single Level II study and single Level III study).

Lumbar medial nerve ablation is suggested for the short-term (3- to 6-month) relief of facet-mediated pain in patients who have chronic lower-back pain without radiculopathy from degenerative disease of the lumbar spine (4 Level II studies).

\section{Lumbar Trigger Point Injections}

\section{Grade B}

Trigger point injections (TPIs) performed as dry needling, with anesthetics alone or with steroids, are not recommended in patients with chronic low-back pain without radiculopathy from degenerative disease of the lumbar spine because a long-lasting benefit has not been demonstrated (Level II evidence).

\section{Diagnostic Recommendations}

\section{Grade B}

To establish the diagnosis of lumbar facet-mediated pain, the double-injection technique with an improvement threshold of $80 \%$ or greater is suggested (single Level I study).

Grade C

Diagnostic facet blocks by the double-injection technique with an improvement threshold of $80 \%$ are an option for predicting a favorable response to facet medial nerve ablation by thermocoagulation for facet-mediated chronic low-back pain without radiculopathy in patients with degenerative disease of the lumbar spine (single Level II study).

\section{Grade I: Inconclusive}

There is no evidence to support the use of diagnostic facet blocks as a predictor of lumbar fusion outcome in patients with chronic low-back pain from degenerative lumbar disease (conflicting Level IV evidence).

\section{Rationale}

Since the original publication of the Lumbar Fusion Guidelines, injection techniques using an anesthetic agent, typically in combination with a steroid, continue to be widely used in the treatment of patients with chronic low-back pain. ${ }^{27}$ An updated analysis of the literature regarding these treatments was performed from July 2003 , the termination point of the previous guidelines, through the end of 2011. As was the case in the original guidelines, an attempt was made to answer 3 questions:

1) Are lumbar ESIs effective for improving the outcomes of patients with chronic low-back pain resulting from degenerative disease of the lumbar spine?

2) Are lumbar facet injections effective for improving the outcomes of patients with chronic low-back pain resulting from degenerative disease of the lumbar spine?
3) Are lumbar TPIs effective for improving the outcomes of patients with chronic low-back pain resulting from degenerative disease of the lumbar spine?

\section{Search Criteria}

A computerized search of articles published from July 2003 through the year 2011 in the National Library of Medicine's MEDLINE database was conducted using the online search engine "PubMed." The search chain included the following terms: "low back pain" [MeSH Terms] OR ("low"[All Fields] AND "back"[All Fields] AND “pain”[All Fields]) OR “low back pain”[All Fields]) AND ("Injections, Spinal" [MeSH] OR "Injections, IntraArticular"'[MeSH] OR “Anesthesia, Epidural"'[MeSH] OR "Nerve Block" [MeSH] OR trigger point injection [title] OR trigger point injections[title] OR (facet joint injection[title] OR facet joint injections[title] OR (epidural steroid injection[title] OR epidural steroid injections [title]) OR epidural steroid block[title] OR (caudal injection[title] OR caudal injections[title]) OR (caudal block[title] OR caudal blockade[title] OR caudal blocks [title]) OR (selective nerve root injection[title] OR selective nerve root injections[title]) OR (selective nerve root block[title] OR selective nerve root blocks[title]) OR (transforaminal injection[title] OR transforaminal injections[title] OR (transforaminal block[title] OR transforaminal blocks[title])) OR (block[title] OR block/ activation[title] OR block/cytological[title] OR block/intra osseous[title] OR block/mylohyoid[title] OR block/neu rolysis[title] OR block/sick[title] OR block/western[title] OR block'[title] OR block's[title] OR block98[title] OR blockable[title] OR blockad[title] OR blockada[title] OR blockade[title] OR blockade/myosin[title] OR block ade/thiazide[title] OR blockade'[title] OR blockaded [title] OR blockaden[title] OR blockader[title] OR block aders[title] OR blockaders/admin[title] OR blockades [title] OR blockading[title] OR blockador[title] OR block age[title] OR blockages[title] OR blockain[title] OR blockaine[title] OR blockal[title] OR blockase[title] OR blockboard[title] OR blockbuilding[title] OR block buster[title] OR blockbuster'[title] OR blockbusters[title] OR blockcourse[title] OR blockcycler[title] OR block dissection[title] OR blocke[title] OR blocked[title] OR blocked'[title] OR blocker[title] OR blocker/5[title] OR blocker/beta[title] OR blocker/calcium[title] OR blocker/ carbonic[title] OR blocker/diuretic[title] OR blocker/drug [title] OR blocker/hydrochlorothiazide[title] OR blocker/ statin[title] OR blocker/thiazide[title] OR blocker/vaso dilator[title] OR blocker's[title] OR blockerette[title] OR blockers[title] OR blockers/ace[title] OR blockers'[title] OR blockes[title] OR blockexcision[title] OR blockface [title] OR blockheads[title] OR blockholer[title] OR blocki[title] OR blockinducing[title] OR blockiness[title] OR blocking[title] OR blocking/deblocking[title] OR blocking/diuretic[title] OR blocking/percolation[title] OR blocking/unblocking[title] OR blocking'[title] OR block ings[title] OR blocklength[title] OR blockley[title] OR blockmakers[title] OR blockmaking[title] OR blockmilk [title] OR blockout[title] OR blockpnea[title] OR block polymer[title] OR blocks[title] OR blocks'[title] OR block sequences[title] OR blockset[title] OR blocksom[title] OR 
blockwise[title] OR blockwriting[title] OR blocky[title] OR blockypnea[title] OR blockzone[title]) OR (facet joint block[All Fields] OR facet joint blocks[All Fields]) OR (median nerve block[title] OR median nerve block ade[title]) OR median nerve injection[title] OR (trigger point injection[title] OR trigger point injections[title]) OR (trigger[All Fields] AND (point block[title] OR point blocks[title])))) AND ((“'Lumbosacral Region”[MeSH] OR "Lumbar Vertebrae"[MeSH]) OR lumbar[title]) AND ((“2003”[PDAT]: “3000”[PDAT]) AND "humans" [MeSH Terms] AND English[lang]).

The search was limited to English-language publications and human subjects. Nonsystematic reviews were discarded, but the bibliographies from these papers were searched for any additional relevant references. The search yielded 249 new references for this paper. Papers selected were confined to studies of chronic low-back pain (>3-6 months) due to lumbar degenerative disease without deformity and without radiculopathy. The results of the search were divided into 3 categories depending on the type of injection investigated: ESIs, facet injections, and TPIs. All papers providing Level II or better evidence were included. In the absence of Level 1 or Level II data, Level III papers were included in the analysis. Papers with Level IV evidence were referenced in the discussion but not included in the evidentiary tables.

\section{Scientific Foundation}

Use of Lumbar ESIs (Interlaminar Injections, Caudal Injections, Transforaminal Injections) in the Treatment of Chronic Low-Back Pain Due to Degenerative Disease of the Lumbar Spine

Epidural injections continue to be used extensively in the treatment of spinal pain. ${ }^{11,34}$ The evaluation of ESIs for chronic lower-back pain without radiculopathy remains minimal. In the previous review of this topic, ${ }^{27} 4$ randomized control trials (RCTs) were found to evaluate the effectiveness of epidural injections in the treatment of chronic lower-back pain., ${ }^{4,9,28,30}$ All 4 of these studies were reported as RCTs but were greatly underpowered and represented equivalence trials without true control groups. By the criteria of the current report, these studies are classified as Level III data and give little support for the use of lumbar ESIs in chronic back pain for anything more than short-term relief $(<2$ weeks). They are referenced in the bibliography but not in the evidence table (see Table 1). Since the completion of the previous review of this topic, a prospective cohort study published in 2004 by Buttermann evaluated 232 patients, age 18-65 years, with low-back pain of greater than 1 year in duration, in whom conservative maneuvers failed. 5 The patients were diagnosed with degenerative disc disease without stenosis or listhesis. They received 1-3 interlaminar or transforaminal steroid injections guided by fluoroscopy and were followed up for up to 2 years. Modic endplate changes on MRI, indicative of vertebral inflammation, were observed in 93 of the study participants. Buttermann predicted that these participants would appreciate more frequent relief of low-back pain after ESIs than would the group with- out inflammatory changes. Validated outcome measures were used, including the visual analog scale (VAS), Oswestry Disability Index (ODI), and pain drawings. Medication usage and the degree of patient satisfaction were also recorded. A subgroup of patients was randomized to receive a discogram with or without steroids. For patients with inflammatory endplate changes, $55 \%$ were satisfied with the degree of pain relief up to 3 months after the injection, although a clinically relevant improvement was not observed in the VAS or ODI scores. A similar finding was observed in the noninflammatory cohort with $47 \%$ satisfied. Improvement in both groups declined over time. While the baseline differences in ODI scores between the two groups was not different prior to treatment, comparison of these scores for the two groups at 3-and 6-month follow-up showed a statistically greater improvement for the group with inflammatory changes $(p<0.001)$, though neither group demonstrated a statistically significant improvement over baseline scores. This study has been cited as providing support for the short-term benefit of ESIs in decreasing chronic low-back pain, although there was no objective improvement in either group observed with the validated outcome measures. Furthermore, the conclusions of the study are severely compromised by the high dropout rate at final follow-up: $51 \%$ of the original patients in the inflammatory group and $60 \%$ of those in the noninflammatory cohort were lost to follow-up. In an equivalence trial, Manchikanti et al. published a randomized controlled and double-blinded study of 70 patients with lower-back pain and no radiculopathy or evidence of disc herniation on MRI. ${ }^{19}$ Thirty-five of the patients were randomized to Group I in which the patients received interlaminar injections of anesthetic only and 35 were randomized to Group II in which the patients received interlaminar injections of an anesthetic and a steroid. Validated outcomes measures, including the ODI for functional assessment and the Numerical Rating Scale (NRS), were recorded at baseline and at 3, 6, and 12 months. Greater than a $50 \%$ improvement in pain or function from baseline was required for significance. Significant pain relief was recorded in 74\% of Group I and $63 \%$ of Group II, while significant functional improvement was achieved in $71 \%$ of Group I and $60 \%$ of Group II. The overall average number of injections for the two groups over the year of follow-up was 4 . This study, while suggestive, suffers from being an equivalence study without appropriate placebo control, from being underpowered, and from being a preliminary report.

Three systematic reviews were identified during the current search (Table 1). Abdi et al. performed a review of the literature from published 1966 to 2006 on cervical, thoracic and lumbar ESIs. ${ }^{1}$ For the lumbar spine, 13 randomized control trials (RCTs) studies for transforaminal injections, and 8 RCTs and 5 prospective trials for caudal injections. The majority of these studies investigated the utility of these treatments for radiculopathy. With respect to chronic low-back pain, the Buttermann study, reviewed above, was felt to provide indeterminate evidence that ESIs were effective in managing chronic low-back pain when the transforaminal and interlaminar techniques were used. ${ }^{5}$ In addition, the authors concluded there was 
W. C. Watters III et al.

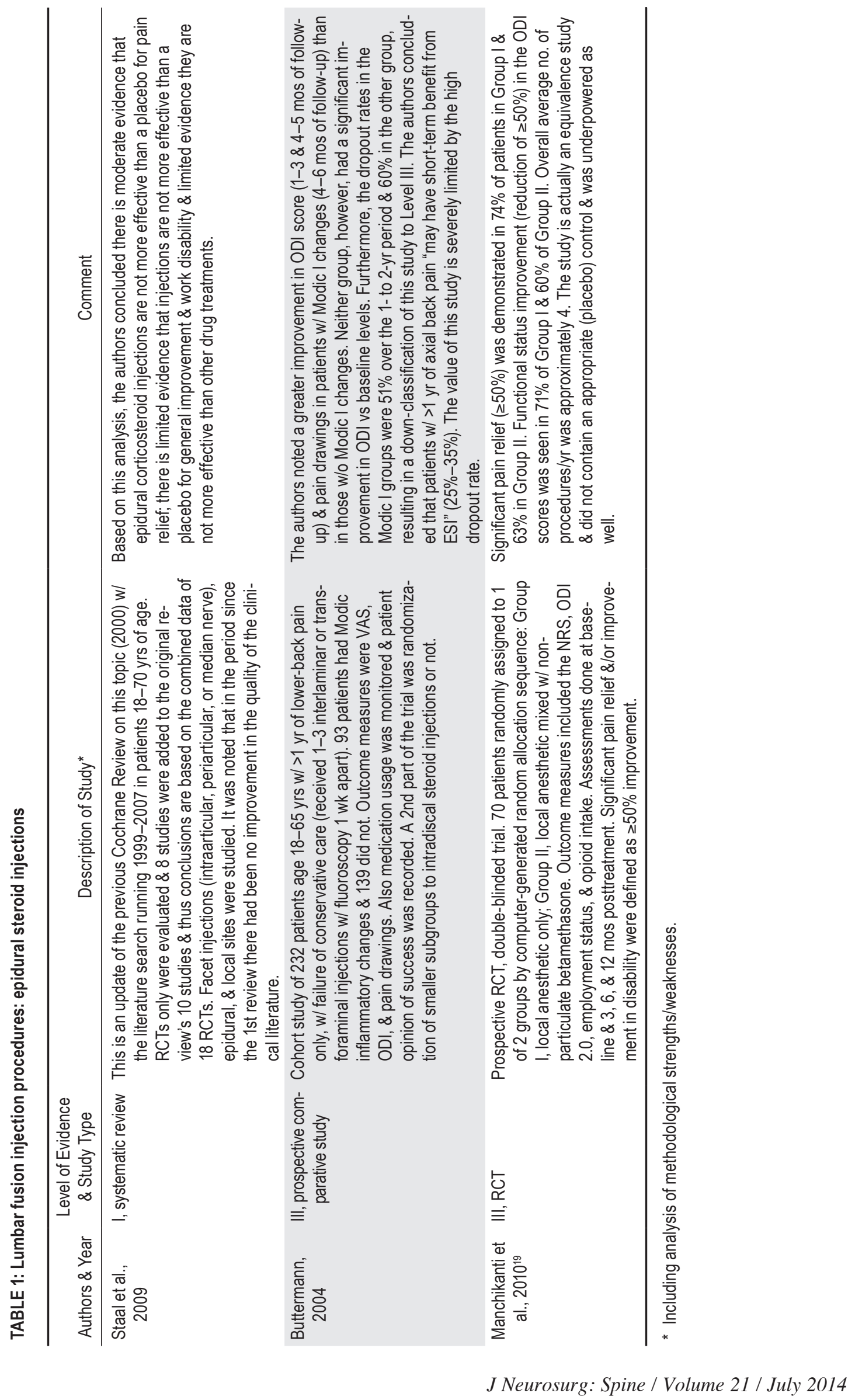


moderate evidence in support of short- and long-term improvement in managing chronic low-back pain via the caudal approach. In 2009, Staal et al. published an update of a previous Cochrane Review, evaluating the literature from 1999 to March 2007 in patients 18-70 years of age..$^{32}$ Only RCTs involving facet, epidural, and local injections were considered. The authors noted that since their initial publication in $2001,{ }^{25}$ there was no apparent improvement in the quality of the evidence. With respect to ESIs for chronic low-back pain, the authors concluded that there was moderate evidence that ESIs are no more effective than a placebo for pain relief, that there is limited evidence ESIs and placebo are equally effective for general improvement in the work-disability population, and that there is limited evidence ESIs are more effective than other drug treatments. Parr et al. published a systematic review of studies published between 1966 and 2008 on lumbar interlaminar injections for the management of chronic low-back pain with and without radiculopathy. ${ }^{26}$ They noted that the majority of these studies were done without fluoroscopic guidance. None of the RCTs identified investigated chronic low-back pain in the absence of a radiculopathy, and of the 30 observational studies, only the Buttermann article evaluated patients with isolated chronic low-back pain. ${ }^{5}$ The authors concluded that the Buttermann article suggested some short-term but no long-term effect for ESIs on chronic lower-back pain.

\section{Use of Lumbar Facet Injections for Chronic Low-Back Pain Due to Degenerative Disease of the Lumbar Spine}

Lumbar facet (zygapophysial) joint injections have been used for both the diagnosis and treatment of facetmediated low-back pain. Facet-mediated pain patterns have been explored by mapping the response to facet provocation and anesthesia injections in volunteers. These studies have yet to demonstrate a reliable pattern of pain produced by an injection within a particular lumbar facet joint. ${ }^{7}$ When the data are combined from multiple studies, patterns emerge that suggest there is considerable overlap among all lumbar facet joints. Pain from the lower facet joints can be referred to the groin and deep posterior thigh, while the upper joints can lead to pain in the flank, hip, and upper lateral thigh. Pain referred below the knee is highly questionable. No physical or radiographic findings consistently correlate with the observations following facet blocks, ${ }^{7}$ and the diagnosis of facet-mediated pain continues to rely on appropriately performed diagnostic facet blocks. The results of so-called double-block studies suggest that facet-mediated low-back pain is a cause of chronic pain in $9 \%-42 \%$ of patients with degenerative lumbar disease. . $^{27,10,18,29}$

Studies investigating the role of diagnostic facet joint blocks have been conducted in an attempt to improve the accuracy of this technique. Since the original guideline publication, a more uniform definition of a valid response has been adopted. It has been suggested that the doubleblock technique is the most reliable means of identifying facet-mediated pain, although this procedure is rarely performed during routine clinical practice. In the doubleblock technique, facet blocks are performed on two different dates with anesthetics that vary with respect to du- ration of the analgesic effect. A positive response requires that the patient's low-back pain significantly improve following both blocks for a period of time consistent with the anesthetic's duration of action. ${ }^{2}$ To further refine the specificity of diagnostic facet injections, it has been suggested that the traditional threshold of greater than $50 \%$ pain relief be increased to greater than $80 \%$. In a systematic review of 7 studies, Datta et al. presented Level I and II diagnostic evidence that the use of double controlled blocks and an $80 \%$ pain relief threshold produced the highest specificity in diagnosing facet-mediated back pain (Table 2)..$^{10}$ They recommended that all future systematic reviews and investigations use these parameters as valid criteria to diagnose facet-mediated pain and evaluate the response to treatment. In an observational study, Manchikanti et al. demonstrated the improved sustainability of the diagnosis of lumbar facet-mediated pain at 2-year follow-up when comparing a group in which the $80 \%$ threshold was used for diagnosis and a group in which a $50 \%$ threshold for pain relief was used for diagnosis. ${ }^{20}$ The diagnosis of facet-mediated pain was sustained in $89.5 \%$ of the patients diagnosed with the double-injection technique and an $80 \%$ threshold at 2 years versus only $51 \%$ of patients diagnosed with a double injection technique and a $50 \%$ threshold. The authors point out that utilizing the double-injection technique and an $80 \%$ threshold will diminish inappropriate and unnecessary treatment. ${ }^{20}$

Therapeutic facet blocks can be delivered in one of two manners: as an intraarticular injection into a facet joint or as a neural block of the medial nerve that innervates the facet capsule. In the previous review of this topic, 3 Level II studies addressed the efficacy of intraarticular injections in the facet joint. ${ }^{6,17,23}$ Lilius et al. randomly assigned patients to one of three groups. ${ }^{17}$ Group I received an intrafacet injection of steroid and anesthetic; Group II, pericapsular injections of steroid and anesthetic; and Group III, pericapsular injections of saline. The authors concluded that facet injections were a nonspecific form of treatment of lower-back pain that had good results depending more on psychosocial aspects of back pain. Carette et al. randomized 91 patients to facet injections of either methylprednisolone or saline. ${ }^{6}$ No differences were seen between the groups at 1, 3, and 6 months postinjection. The authors concluded that injection of methylprednisolone into facet joints was of little treatment value. Marks et al. randomized 86 patients with chronic lower-back pain to receive either a facet injection with steroid and anesthetic or just an anesthetic block of the joint. ${ }^{23}$ They concluded that at 3 months both types of injections were equally good diagnostically and equally unsatisfactory for treatment of chronic lower-back pain. The additional literature reviewed for the current report suggests that there is little evidence supporting the value of intraarticular facet blocks as a therapeutic option for chronic low-back pain, prompting one investigator to comment that the efficacy of these injections was no greater than a sham injection. ${ }^{3}$ Datta et al. ${ }^{10}$ performed a systematic, evidence-based review of the literature from 1966 through 2008 and identified 1438 articles investigating the utility of lumbar facet injections. They excluded 
W. C. Watters III et al.

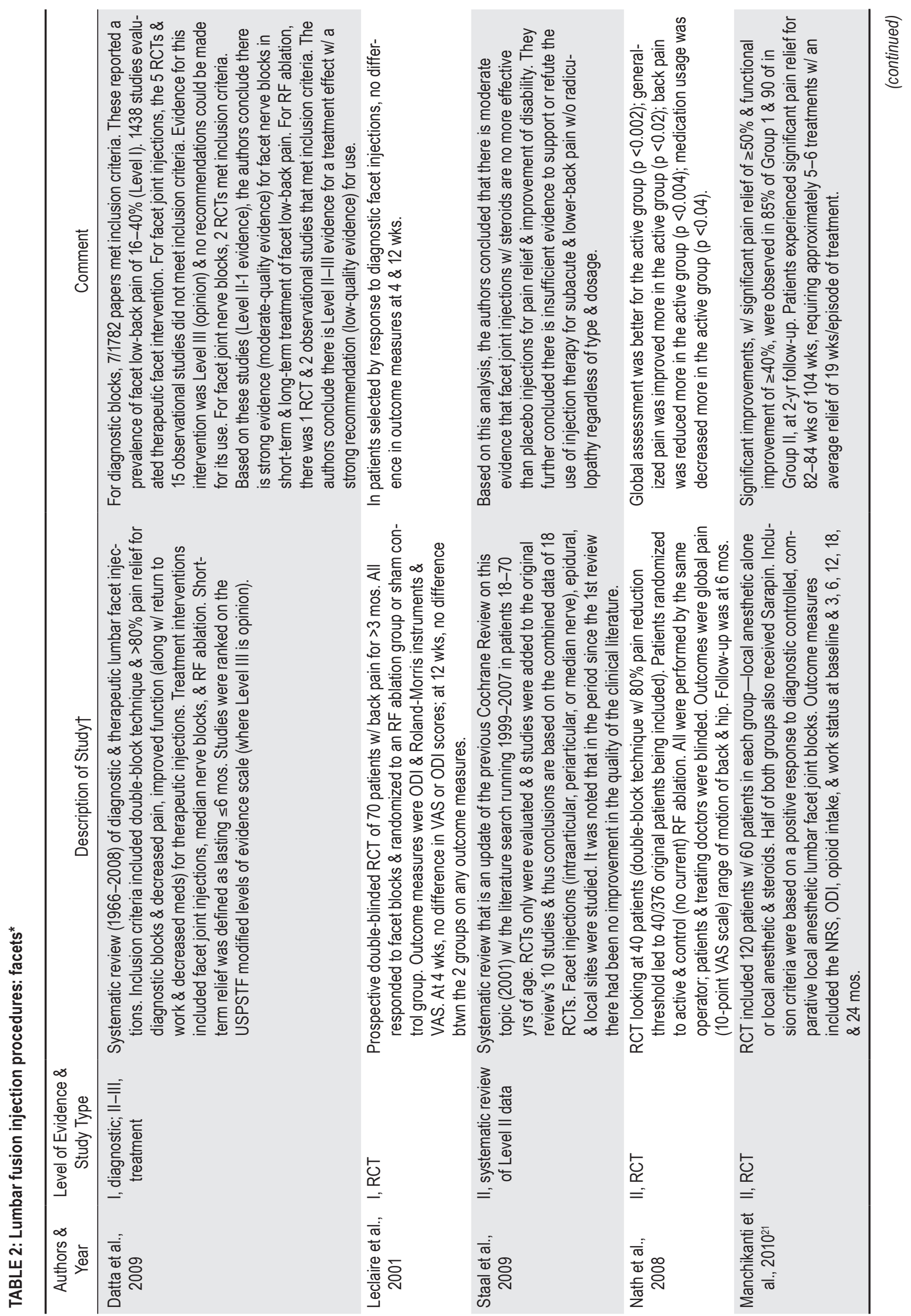


Part 13: Injection therapies, low-back pain, and lumbar fusion

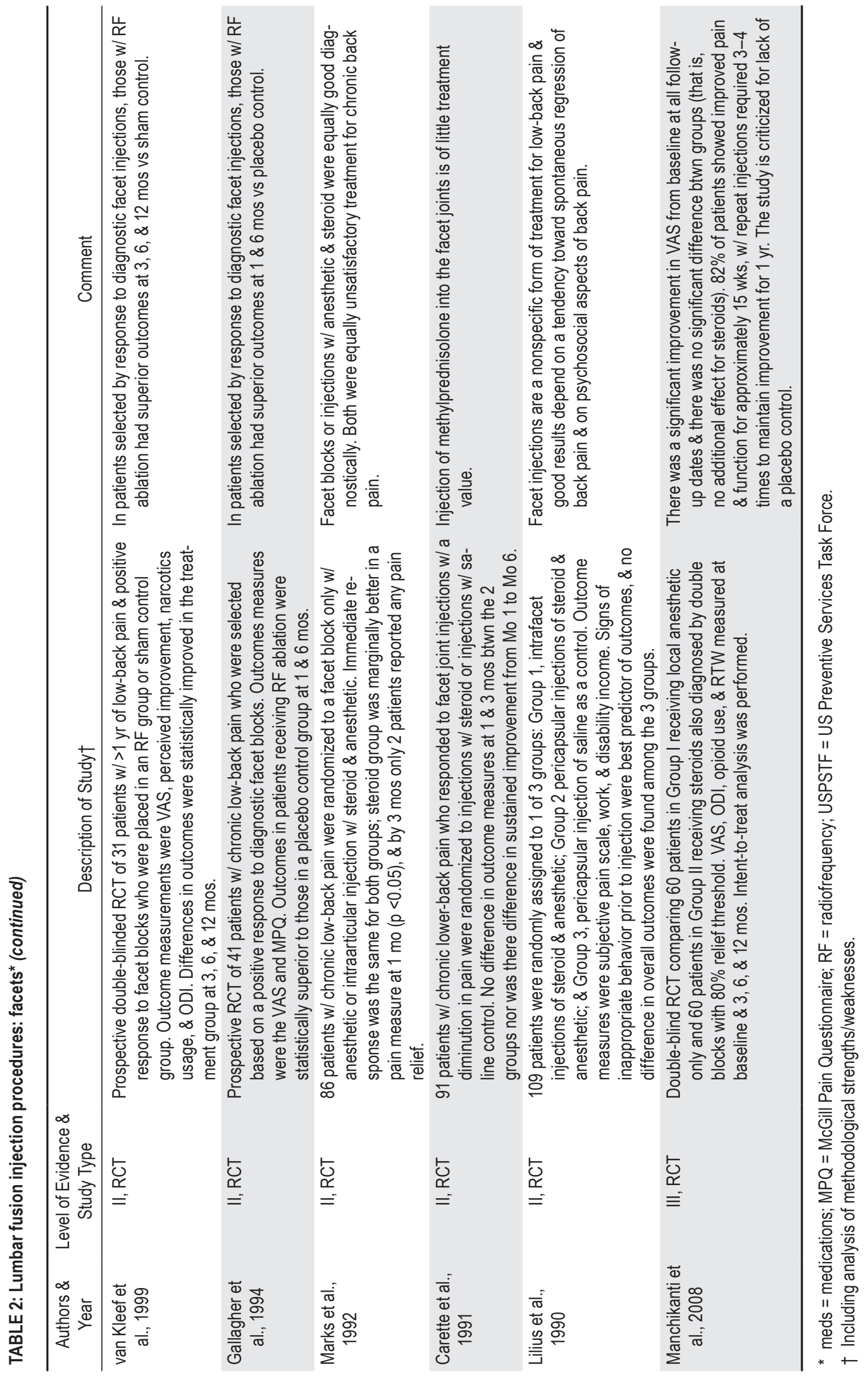


studies not evaluating patients with chronic low-back pain of more than 3 months' duration that was diagnosed as facet-mediated pain by the double-injection technique, with a greater than $80 \%$ pain relief threshold. Six RCTs and 15 observational studies were identified that evaluated the effectiveness of lumbar intraarticular facet injections. These studies were rejected due to poor methodology and failure to use the double-injection technique to confirm the diagnosis. Based on this systematic review of low-quality evidence, the authors concluded that there was no role for intraarticular facet injections as a treatment modality. This conclusion was supported by the update of the Cochrane Review published by Staal et al. in 2009. ${ }^{32}$ These authors identified moderate-level evidence that facet joint injections with steroids are no more effective than placebo injections for relief of pain and disability.

The evidence for therapeutic efficacy is better for medial nerve blocks of the lumbar facet joint. In their systematic review, by Datta et al. also evaluated the role of lumbar facet nerve blocks as a therapeutic intervention. ${ }^{10}$ They identified two RCTs that met inclusion criteria but no observational studies. Manchikanti et al. performed a double-blinded RCT of 120 patients with facet-mediated low-back pain of greater than 6 months' duration diagnosed using the double-injection technique and an $80 \%$ relief threshold. ${ }^{22}$ All patients underwent a fluoroscopically guided injection of the medial nerve. Group I ( $n=$ $60)$ received anesthetic only and Group II $(n=60)$ received anesthetic and steroid. Half of each group also received Sarapin in the injectant. Multiple injections were performed at the discretion of the treating physician over 1 year. Validated outcome measures including the VAS and ODI were used along with nonvalidated measures of drug usage and return-to-work status. An intent-to-treat analysis was used to evaluate the data at final followup. Patients received up to 5 injections over the 1 -year period with an average of 3.4 injections per patient. Improved pain scores, with over $50 \%$ pain relief reported in over $80 \%$ of the participants, were observed at 3,6 , and 12 months after the first injection when compared with baseline; however, no differences were observed between treatment groups. The ODI results were also significantly improved at 3,6, and 12 months in all groups but with no differences between treatment groups. There was no significant decrease in opioid use observed in any group. These results support the premise that patients may experience significant pain relief from multiple injections for up to 44-45 weeks, with each injection providing on average of 15 weeks of pain relief for low-back pain and increased function as measured by the ODI. This study, an equivalence study that did not include a placebo control, provides moderate evidence that medial nerve injections confer short-term relief of chronic facet-mediated low-back pain. In a 2-year follow-up study of this same group of patients, Manchikanti et al. demonstrated that outcomes were sustained in both groups..$^{21}$ Pain relief of greater than $50 \%$ and functional improvement of greater than $40 \%$ were seen in $85 \%$ of Group I and $90 \%$ of Group II at 18 and 24 months. Continued need for repeated injections, with an average of 5 or 6 injections over the study period and duration of effect of 19 weeks, was seen in the longer follow-up. ${ }^{21}$

Ablation of the medial nerve, through radiofrequency thermocoagulation, is a variant of the facet nerve block. In the previous review of this topic, several papers were found testing the ability of facet blocks to predict outcomes from radiofrequency thermocoagulation. ${ }^{27}$ Gallagher et al. performed a prospective, double-blinded RCT on 41 patients who reported either a strong or equivocal response to diagnostic facet blocks. ${ }^{13}$ These 41 patients received either radiofrequency ablation with an anesthetic or just an anesthetic injection. Outcomes were assessed using the McGill Pain Questionnaire and VAS at 1 and 6 months. Patients who were strongly positive on facet blocks and received radiofrequency ablation did statistically better on both outcome measures at both times than those who were poor responders to facet blocks and received ablation. Van Kleef et al. randomized 31 patients who had responded strongly to facet blocks into two groups: one received radiofrequency ablation and the other received a sham control. ${ }^{33}$ Both patients and treating doctors were blinded as to treatment who was in the control group. Outcomes were assessed using the VAS and ODI and by quantification of the amount of narcotic used. Outcomes were statistically superior in the radiofrequency group over the control at 3, 6, and 12 months. In a larger blinded RCT of 70 patients who had responded to facet blocks, Leclaire et al. measured outcomes after radiofrequency ablation using the VAS, ODI, and Roland-Morris disability questionnaire and found that results were superior only at 2 weeks, indicating no superiority for radiofrequency ablation for long-term relief of lower-back pain in this study. ${ }^{16}$ Nath et al. conducted a randomized, double-blinded study of patients with chronic low-back pain of 2 years' duration in whom conservative treatment failed. ${ }^{24}$ They included only patients with facet-mediated low-back pain, diagnosed by the double-block technique and a threshold of greater than $80 \%$ pain reduction. From a potential population of 376 candidates, only 40 patients fulfilled all the diagnostic criteria. These patients were randomized into a treatment group $(n=20)$, receiving active radiofrequency ablation, and a placebo group $(n=20)$, undergoing an identical sham procedure. Primary outcome measures included a VAS pain scale and a nonvalidated, self-reported 1- to 6-point global improvement scale. Lumbar range of motion and a 6-point quality of life scale were used as secondary outcome measures. Generalized pain, low-back pain, and referred pain were all significantly reduced in the treatment group compared with the control group at 6 months' follow-up. Although this is an underpowered study, the strict diagnostic inclusion criteria lend strength to its conclusions. This paper provides moderate evidence for the effectiveness of facet radiofrequency ablation in the short-term treatment of facet-mediated back pain.

Despite the increased diagnostic rigor seen more frequently in the newer literature (the double blocks and the $80 \%$ threshold for pain reduction), no new studies have appeared to suggest that diagnostic facet blocks can effectively predict the outcomes of surgical fusion in patients with chronic low-back pain from lumbar degenerative disease. 
Use of Local Lumbar Injections (TPIs) in the Treatment of Chronic Low-Back Pain Due to Degenerative Disease of the Lumbar Spine

In the previous review of TPIs for chronic low-back pain, 4 Level II RCTs of small patient numbers were presented..$^{8,14,15,31}$ In a very small study, Hameroff et al. randomized, in a double-blind fashion, 15 patients into 3 groups: Group 1 received bupivacaine TPIs, Groups 2 received etidocaine injections, and Group 3 received a saline control injection. ${ }^{15}$ Subjective reports of pain were obtained at 15 minutes, 1 day, and 7 days after injection. Trigger point injections with anesthetic were more effective than those with saline. Sonne et al. ${ }^{31}$ prospectively randomized 30 patients with at least 1 month of lowerback pain into 2 groups in a double-blinded study: Group I received an injection of methylprednisolone with lignocaine and Group II received an injection of isotonic saline. Outcome measures were the VAS and lumbar range of motion. Significant decreases in VAS scores were seen in the anesthesia/steroid group while there was no difference between the two groups in terms of range of motion. Garvey et al. performed a randomized, double-blind evaluation of 63 patients with low-back pain unresponsive to 4 weeks of conservative care. ${ }^{14} \mathrm{He}$ divided the patients into 4 groups: Group I was treated with lidocaine TPIs, Group II with lidocaine and steroid TPIs, Group III with dry needling, and Group IV with acupressure and vapocoolant. More patients reported decreased pain in response to acupressure and coolant $(63 \%)$ than to drug TPIs (42\%), but the difference was not significant. The authors concluded that TPIs have some potential value in treating lower-back pain but that injecting a drug was not necessary. Collée et al., in a double-blind study, randomly assigned 41 patients to receive TPIs with $0.5 \%$ lignocaine or saline. ${ }^{8}$ Outcome measures were the VAS and a painintensity scores measured 2 weeks after injection. The group receiving the anesthetic had a significantly better decrease in pain than did the saline group. For all of these studies, it should be noted that none of the patient groups fulfilled a definition of chronic lower-back pain (>3 months' duration). In reviewing the literature for the current review, no high-quality studies on the efficacy of TPIs were found since the original Guideline publication. There have, however, been 2 published systematic reviews that focused partially or completely on TPIs (see Table 3).

In 2005 Furlan et al. published a Cochrane Review focusing on acupuncture and dry-needling for both acute and chronic low-back pain and reviewed the literature from 1996 to February 2003. ${ }^{12}$ While 35 RCTs were identified, only 20 of these were in English and all of the RCTs were felt to have significant methodological flaws. With respect to dry needling for chronic low-back pain, the authors concluded that the evidence was insufficient and of exceedingly poor quality to formulate any meaningful recommendations. A more contemporary Cochrane Review of injection therapy for subacute and chronic low-back pain by Staal et al. included TPIs as a treatment alternative for chronic low-back pain patients. ${ }^{32}$ The literature published between 1999 and 2007 was reviewed. The authors concluded, based on limited data, that TPIs with steroids are no more effective than pla- cebo injections for pain relief and improvement of disability. They stated that there was insufficient evidence to support the use of injection therapy for subacute and chronic low-back pain without radiculopathy regardless of type and dosage. The studies reviewed in the original Guidelines as well as these 2 systematic reviews suggest no significant differences in treatment effect exist among the uses of an anesthetic, an anesthetic and steroid, or dry needling with TPIs. Any improvement seen with these techniques was only apparent in acute cases of low-back pain. No evidence was available to support the effectiveness of TPIs in the treatment of chronic low-back pain.

\section{Summary}

Based on the literature reviewed for the original guideline publication as well as this updated review, there is weak evidence that ESIs provide short-term relief of pain in patients with chronic low-back pain from degenerative lumbar disease. There is evidence that caudal ESIs are an option for decreasing pain for greater than 6 weeks in patients with chronic low-back pain from degenerative lumbar disease (Level III evidence).

Based on the original guidelines as well as this updated review, there is moderate evidence to recommend that the diagnosis of facet-mediated back pain be made with the double-injection technique and a greater than $80 \%$ improvement threshold (Level II evidence). There is moderate evidence supporting a recommendation that diagnostic facet blocks be used to predict a good response to facet medial nerve ablation by thermocoagulation for facet-mediated chronic low-back pain (Level II evidence). There is moderate evidence suggesting that there is no role for intraarticular facet injections in the treatment of chronic low-back pain from lumbar degenerative disease (Level II evidence against). There is moderate evidence supporting the use of facet medial nerve blocks to achieve short-term pain relief for patients with facet-mediated chronic low-back pain from degenerative lumbar disease (Level II evidence). There is moderate evidence that facet medial nerve ablation produces a short-term decrease (3-6 months) of facet-mediated chronic low-back pain (Level II evidence).

There is no evidence to support a recommendation that diagnostic blocks are useful predictors of surgical outcomes following lumbar fusion.

Based on the original guidelines as well as this updated literature review, there is no evidence to support the use of TPIs with a dry-needling technique, with anesthetics alone or accompanied by steroids, in the management of patients suffering from chronic low-back pain secondary to degenerative lumbar disease (Level IV evidence).

\section{Acknowledgments}

We would like to acknowledge the AANS/CNS Joint Guidelines Committee (JGC) for their review, comments, and suggestions; Laura Mitchell, CNS Guidelines Project Manager, for her organizational assistance; and Linda O'Dwyer, medical librarian, for assistance with the literature searches. We would also like to acknowledge the following individual JGC members for their contributions throughout the review process: Timothy Ryken, M.D.; 
W. C. Watters III et al.

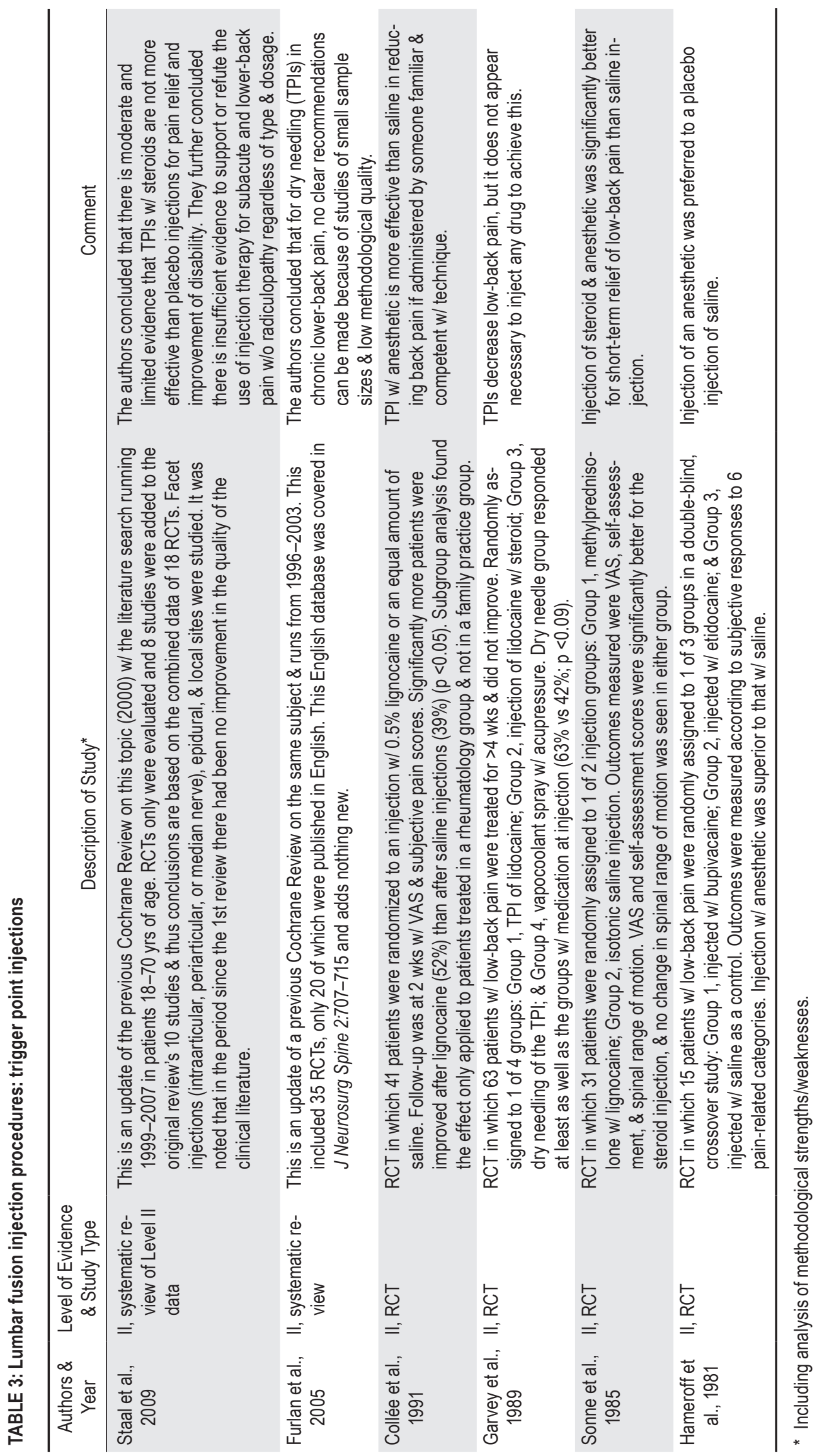


Part 13: Injection therapies, low-back pain, and lumbar fusion

Kevin Cockroft, M.D.; Sepideh Amin-Hanjani, M.D.; Steven N. Kalkanis, M.D.; John O'Toole, M.D., M.S.; Steven Casha, M.D., Ph.D.; Aaron Filler, M.D., Ph.D., F.R.C.S.; Daniel Hoh, M.D.; Steven Hwang, M.D.; Todd McCall, M.D.; Jeffrey J. Olson, M.D.; Julie Pilitsis, M.D., Ph.D.; Joshua Rosenow, M.D.; and Christopher Winfree, M.D.

\section{Disclosure}

Administrative costs of this project were funded by the Congress of Neurological Surgeons and the Joint Section on Disorders of the Spine and Peripheral Nerves of the American Association of Neurological Surgeons and Congress of Neurological Surgeons. No author received payment or honorarium for time devoted to this project. Dr. Ghogawala receives grants from the Patient Centered Outcomes Research Institute (PCORI) and the National Institutes of Health (NIH). Dr. Groff is a consultant for DePuy Spine and EBI Spine. Dr. Mummaneni owns stock in Spinicity and receives honoraria from DePuy Spine and Globus and royalties from DePuy Spine, Quality Medical Publishers, and Thieme Publishing. Dr. Wang owns stock in Bone Biologics, AxioMed, Amedica, CoreSpine, Expanding Orthopedics, Pioneer, Syndicom, VG Innovations, PearlDiver, Flexuspine, Axis, FzioMed, Benvenue, Promethean, Nexgen, ElectroCore, and Surgitech and holds patents with and receives royalties from Biomet, Stryker, SeaSpine, Aesculap, Osprey, Amedica, Synthes, and Alphatec. The authors report no other potential conflicts of interest concerning the materials or methods used in this study or the findings specified in this paper.

Author contributions to the study and manuscript preparation include the following. Acquisition of data: all authors. Analysis and interpretation of data: all authors. Drafting the article: Watters. Critically revising the article: all authors. Reviewed submitted version of manuscript: all authors. Approved the final version of the manuscript on behalf of all authors: Watters. Study supervision: Kaiser.

\section{References}

1. Abdi S, Datta S, Trescot AM, Schultz DM, Adlaka R, Atluri $\mathrm{SL}$, et al: Epidural steroids in the management of chronic spinal pain: a systematic review. Pain Physician 10:185-212, 2007

2. Bogduk N: Evidence-informed management of chronic low back pain with facet injections and radiofrequency neurotomy. Spine J 8:56-64, 2008

3. Bogduk N: A narrative review of intra-articular corticosteroid injections for low back pain. Pain Med 6:287-296, 2005

4. Breivik H, Helsa PE, Molnar I, Lind B: Treatment of chronic low back pain and sciatica. Comparison of caudal injections of bupivacaine and methylprednisolone with bupivacaine followed by saline. Adv Pain Res Ther 1:927-932, 1976

5. Buttermann GR: Treatment of lumbar disc herniation: epidural steroid injection compared with discectomy. A prospective, randomized study. J Bone Joint Surg Am 86-A:670-679, 2004

6. Carette S, Marcoux S, Truchon R, Grondin C, Gagnon J, Allard $\mathrm{Y}$, et al: A controlled trial of corticosteroid injections into facet joints for chronic low back pain. N Engl J Med 325:10021007, 1991

7. Cohen SP, Raja SN: Pathogenesis, diagnosis, and treatment of lumbar zygapophysial (facet) joint pain. Anesthesiology 106: 591-614, 2007

8. Collée G, Dijkmans BA, Vandenbroucke JP, Cats A: Iliac crest pain syndrome in low back pain. A double blind, randomized study of local injection therapy. J Rheumatol 18:1060-1063, 1991

9. Dallas TL, Lin RL, Wu WH, Wolskee P: Epidural morphine and methylprednisolone for low-back pain. Anesthesiology 67:408-411, 1987

10. Datta S, Lee M, Falco FJ, Bryce DA, Hayek SM: Systematic assessment of diagnostic accuracy and therapeutic utility of lumbar facet joint interventions. Pain Physician 12:437-460, 2009

11. DePalma MJ, Slipman CW: Evidence-informed management of chronic low back pain with epidural steroid injections. Spine J 8:45-55, 2008

12. Furlan AD, van Tulder M, Cherkin D, Tsukayama H, Lao L, Koes B, et al: Acupuncture and dry-needling for low back pain: an updated systematic review within the framework of the Cochrane collaboration. Spine (Phila Pa 1976) 30:944-963, 2005

13. Gallagher J, Di Vadi PLP, Wedley JR, Hamann W, Ryan P, Chikanza I, et al: Radiofrequency facet joint denervation in the treatment of low back pain: a prospective controlled double-blind study to assess its efficacy. Pain Clin 7:193-198, 1994

14. Garvey TA, Marks MR, Wiesel SW: A prospective, randomized, double-blind evaluation of trigger-point injection therapy for low-back pain. Spine (Phila Pa 1976) 14:962-964, 1989

15. Hameroff SR Sr, Crago BR, Blitt CD, Womble J, Kanel J: Comparison of bupivacaine, etidocaine, and saline for triggerpoint therapy. Anesth Analg 60:752-755, 1981

16. Leclaire R, Fortin L, Lambert R, Bergeron YM, Rossignol $\mathrm{M}$ : Radiofrequency facet joint denervation in the treatment of low back pain: a placebo-controlled clinical trial to assess efficacy. Spine (Phila Pa 1976) 26:1411-1417, 2001

17. Lilius G, Harilainen A, Laasonen EM, Myllynen P: Chronic unilateral low-back pain. Predictors of outcome of facet joint injections. Spine (Phila Pa 1976) 15:780-782, 1990

18. Manchikanti L, Boswell MV, Singh V, Pampati V, Damron $\mathrm{KS}$, Beyer CD: Prevalence of facet joint pain in chronic spinal pain of cervical, thoracic, and lumbar regions. BMC Musculoskelet Disord 5:15, 2004

19. Manchikanti L, Cash KA, McManus CD, Pampati V, Benyamin RM: Preliminary results of a randomized, double-blind, controlled trial of fluoroscopic lumbar interlaminar epidural injections in managing chronic lumbar discogenic pain without disc herniation or radiculitis. Pain Physician 13:E279E292, 2010

20. Manchikanti L, Pampati S, Cash KA: Making sense of the accuracy of diagnostic lumbar facet joint nerve blocks: an assessment of the implications of 50\% relief, $80 \%$ relief, single block, or controlled diagnostic blocks. Pain Physician 13:133-143, 2010

21. Manchikanti L, Singh V, Falco FJ, Cash KA, Pampati V: Evaluation of lumbar facet joint nerve blocks in managing chronic low back pain: a randomized, double-blind, controlled trial with a 2-year follow-up. Int J Med Sci 7:124-135, 2010

22. Manchikanti L, Singh V, Falco FJE, Cash KA, Pampati V: Lumbar facet joint nerve blocks in managing chronic facet joint pain: one-year follow-up of a randomized, double-blind controlled trial: Clinical Trial NCT00355914. Pain Physician 11:121-132, 2008

23. Marks RC, Houston T, Thulbourne T: Facet joint injection and facet nerve block: a randomised comparison in 86 patients with chronic low back pain. Pain 49:325-328, 1992

24. Nath S, Nath CA, Pettersson K: Percutaneous lumbar zygapophysial (facet) joint neurotomy using radiofrequency current, in the management of chronic low back pain: a randomized double-blind trial. Spine (Phila Pa 1976) 33:1291-1298, 2008

25. Nelemans PJ, deBie RA, deVet HC, Sturmans F: Injection therapy for subacute and chronic benign low back pain. Spine (Phila Pa 1976) 26:501-515, 2001

26. Parr AT, Diwan S, Abdi S: Lumbar interlaminar epidural injections in managing chronic low back and lower extremity pain: a systematic review. Pain Physician 12:163-188, 2009

27. Resnick DK, Choudhri TF, Dailey AT, Groff MW, Khoo L, Matz PG, et al: Guidelines for the performance of fusion procedures for degenerative disease of the lumbar spine. Part 13: 


\section{W. C. Watters III et al.}

injection therapies, low-back pain, and lumbar fusion. J Neurosurg Spine 2:707-715, 2005

28. Rocco AG, Frank E, Kaul AF, Lipson SJ, Gallo JP: Epidural steroids, epidural morphine and epidural steroids combined with morphine in the treatment of post-laminectomy syndrome. Pain 36:297-303, 1989

29. Schwarzer AC, Aprill CN, Derby R, Fortin J, Kine G, Bogduk $\mathrm{N}$ : Clinical features of patients with pain stemming from the lumbar zygapophysial joints. Is the lumbar facet syndrome a clinical entity? Spine (Phila Pa 1976) 19:1132-1137, 1994

30. Serrao JM, Marks RL, Morley SJ, Goodchild CS: Intrathecal midazolam for the treatment of chronic mechanical low back pain: a controlled comparison with epidural steroid in a pilot study. Pain 48:5-12, 1992

31. Sonne M, Christensen K, Hansen SE, Jensen EM: Injection of steroids and local anaesthetics as therapy for low-back pain. Scand J Rheumatol 14:343-345, 1985

32. Staal JB, de Bie RA, de Vet HC, Hildebrandt J, Nelemans P: In- jection therapy for subacute and chronic low back pain: an updated Cochrane review. Spine (Phila Pa 1976) 34:49-59, 2009

33. van Kleef M, Barendse GA, Kessels A, Voets HM, Weber WE, de Lange S: Randomized trial of radiofrequency lumbar facet denervation for chronic low back pain. Spine (Phila Pa 1976) 24:1937-1942, 1999

34. Young IA, Hyman GS, Packia-Raj LN, Cole AJ: The use of lumbar epidural/transforaminal steroids for managing spinal disease. J Am Acad Orthop Surg 15:228-238, 2007

Manuscript submitted March 20, 2014.

Accepted April 9, 2014.

Please include this information when citing this paper: DOI: 10.3171/2014.4.SPINE14281.

Address correspondence to: Michael G. Kaiser, M.D., Columbia University, Neurological Surgery, The Neurological Institute, 710 W. 168th St., New York, NY 10032. email: mgk7@columbia.edu. 